\title{
Visual dimensional dominance and haptic form recognition
}

\author{
CHRISTINE MICALLEF and RICHARD B. MAY \\ University of Victoria, Victoria, British Columbia, Canada
}

\begin{abstract}
Eighty-two preschool children were assessed for dimensional dominance by asking them to make similarities judgments about two dimensional geometric figures varying in form, color, and size. They also participated in two tests of haptic form recognition of three dimensional forms, once with one hand and once with two hands. The results indicate that recognition performance of form dominant subjects was substantially superior to the performance of nonform subjects. Children of mixed dominance improved on the second test, generating a significant type of Dominance by Tests interaction. Order of administration of dominance and haptic tests had no significant influence on recognition performance.
\end{abstract}

Dimensional dominance (Smiley \& Weir, 1966), also called dimensional preference (Seitz \& Weir, 1971), and dimensional salience (Odom \& Guzman, 1972) refer to the consistent tendency of the subject to attend to a particular property of a stimulus such as color, form, or size. It is assumed that there is an internal bias that causes the subject to classify stimuli on the basis of one dimension rather than on others. Typically, preference for a dimension is assessed by means of a single test on which the subject is asked to make similarity judgments about stimuli. The scaling procedure used in most dominance research is the method of triads (Doehring, 1960; Suchman \& Trabasso, 1966). Three stimuli are presented as surface figures or as solids and are arranged at the corners of an undrawn equilateral triangle. The subjects are instructed to point to the two figures that look the same. For example, a subject may be presented a large red circle, a small blue circle, and a large blue triangle and asked, "which two of these things are the same or most alike?" If the subject picks the two circles, he has made a form response; if he picks the two blue figures, a color response; and if he picks the large figures, a size response.

The early dominance assessment work of Brian and Goodenough (1929) demonstrated that the majority of preschool children made more color than form matching responses while the majority of older subjects made more form matching responses. The shift from color to form dominance has been regarded by some investigators as a correlate of cognitive growth as seen, for example, in mental test scores

This research was supported in part by Grant A0342 from the National Research Council of Canada. The authors are indebted to the supervisors and staff of Blue Jay Nursery, B. C. Government Employees Union. Metropolitan United Church, and Jack Horner day care centers for their cooperation and helpfulness during this study. Louis J. Moran sponsors this paper and takes full editorial responsibility for its content. Requests for reprints should be addressed to R. B. May, Department of Psychology. University of Victoria. Victoria, B. C. V8W 2Y2, Canada.
(Brian \& Goodenough, 1929), verbal mediation development (Kagan \& Lemkin, 1961), and discrimination shift performance (Seitz \& Weir, 1971). In these and other cases, form dominant subjects have been regarded as cognitively more sophisticated than nonform subjects. Typically, the relationship between cognitive ability and dimensional dominance was assessed by two very similar tasks. Two dimensional geometric figures were visually presented in the dominance scaling and also in the subsequent problem-solving or related task. If dominance is a good index of general cognitive ability, then it should also predict cross modal performance. Scores obtained by scaling subjects in the visual mode should be good predictors of their performance in the haptic mode.

Support for the hypothesis that haptic form. recognition indicates cognitive growth can be found in a study by Spreen and Gaddes (1969). These investigators used a six-block version of the Seguin Formboard, and subjects were required to draw forms from memory after they explored them haptically. Though different from the present study in purpose, their results demonstrated that older subjects were superior to younger subjects, supporting the utility of haptic recognition as an index of cognitive ability.

In the present study, subjects were assessed for their dominant dimension, either before or after being presented with the haptic form recognition task. A 10-item version of the Seguin Formboard was hidden from view and subjects were asked to haptically explore (actively touch) wooden blocks one at a time and then identify the block by pointing to a corresponding figure of a multiple-choice identification card. On the basis of earlier studies of dimensional dominance, form responders should perform with fewer errors than nonform responders.

The nonform group consists of both color-dominant subjects making the predetermined criterion number of responses on the color dimension and mixed subjects making a number of responses less than the 
criterion on each dimension. Although investigators have not arrived at any conclusive understanding of multidimensional responding, Suchman and Trabasso (1966) have proposed that this type of responding occurs when young subjects are at a temporary transition stage between color and form dominance. Thus it is expected that mixed responders will recognize forms presented haptically with fewer errors than color subjects but with significantly more errors than form subjects.

\section{METHOD}

\section{Subjects}

The subjects were 105 children, 53 boys and 52 girls, drawn from four day care centers in Victoria. B. C. during October, 1974 and February, 1975. In an effort to have form, color, and mixed dominance groups of approximately equal size, 23 children were dropped from the study because form and color dominance was overrepresented in the present sample. The remaining subjects were 41 boys and 41 girls of whom 28 were form dominant, 28 color dominant, and 26 mixed responders. The mean age of these 82 subjects was 4 years 5 months (range $45-62$ months). The mean age of each dominance group and distribution of boys and girls is given in Table 1 .

\section{Stimuli}

Dominance scaling. The stimuli for assessing dimensional dominance were two-dimensional figures presented in triads on $12 \times 17 \mathrm{~cm}$ white cards. Eight stimulus cards were randomly selected from the stimulus set of 24 cards previously used by Fernandez (1973). Each card contained three figures arranged at the corners of an undrawn equilateral triangle. On any given card the figures varied in two values of the dimensions form, color and size. The stimulus values of figures used were: vivid red or dark blue circles and triangles; $1.8-\mathrm{cm}$ vs. $2.8-\mathrm{cm}$-diam circles and $2.2-\mathrm{cm}$ vs. $3.4-\mathrm{cm}$ equilateral triangles. The cards were counterbalanced for position and combinations of values across cards.

Haptic recognition stimuli. Subjects were seated in front of a wooden box-like apparatus which contained a rectangular aperture $40 \mathrm{~cm}$ wide on the lower front side. A black curtain hung over the aperture throughout the experiment to prevent the subjects from observing the objects they were to manipulate. The object stimuli were from the Seguin Formboard used by Spreen and Gaddes (1969). They consisted of 10 wooden three-dimensional forms $2 \mathrm{~cm}$ thick (rectangle, square, star, cross, and irregular shapes), with average size of $11 \times 9 \mathrm{~cm}$. Each of the stimuli could be matched with 1 of 10 solid black figures located on a white identification card. This card was $65 \times 45 \mathrm{~cm}$ and fastened to a box-like supporting structure placed to the right of the curtained aperture. The subject had to turn in his chair to have complete vision of the recognition figures. Two identification cards were used throughout the experiment, each with the same figures located in different positions which were randomly selected. The identification card was changed for each subject by either turning the original upside down or exchanging it for the second card. Hence, no two successive subjects were tested for recognition of forms located in the same order.

\section{Procedure}

In scaling dimensional dominance, each child was asked to point to tw' of the three figures which were "the same or most alike." The cards were shuffled between subjects, and no feedback was given. A criterion for dominance for either form, color, or size was set at seven of eight $(87.5 \%$ ) choices of one dimension. This corresponds to a significant choice of a dimension at the .035 level. Subjects making less than seven choices of a single dimension were called mixed responders.
Table 1

\begin{tabular}{|c|c|c|c|}
\hline & \multicolumn{3}{|c|}{ Scaling Classification } \\
\hline & Form & Color & Mixed \\
\hline Mean & 53.7 & 53.6 & 51.9 \\
\hline SD & 4.1 & 3.8 & 3.9 \\
\hline Range & $45-62$ & $48-61$ & $45-60$ \\
\hline Boys & 15 & 15 & 11 \\
\hline Girls & 13 & 13 & 15 \\
\hline Total N & 28 & 28 & 26 \\
\hline
\end{tabular}

Forty-two subjects were scaled for dominance prior to being tested for haptic recognition, while 40 subjects were scaled for dominance after having completed the haptic recognition task. This counterbalance was used since Tighe, Tighe, Waterhouse, and Vasta (1970) demonstrated that prior dominance scaling had an influence on a discrimination task whereas scaling after discrimination training was unrelated to performance. Of the 42 subjects scaled for dominance before the form recognition task, 21 subjects used one hand first to haptically explore the test objects. and 21 subjects used two hands first. However, of the 40 subjects scaled for dominance after the form recognition task, 21 subjects used one hand first while 19 subjects used two hands first. The difference in number of subjects in this latter group was due to the experimenter's inability to obtain two more subjects for the mixed dominance group, when dominance scaling was given after the haptic recognition task.

Before presenting the object stimuli for recognition, each subject was familiarized with the individual black figures located on the identification card. This was done by pointing to each figure and asking the subject, "do you know what this is?" The experimenter tried to make this a game, and, though many subjects gave incorrect responses, they were praised for attempting to identify the black figures. This procedure served to familiarize the subjects with each form and assure the experimenter that all figures on the identification card had been attended to at least once.

Although a set of formal instructions was not read to the subjects before the recognition task, the experimenter introduced the task in such a way as to clearly communicate that the subject was to place his hand or hands behind the curtain and feel the object until he could recognize the corresponding figure. The object was to remain behind the curtain and could not be seen until after the complete game.

The subjects performed haptically by inserting one hand or two hands (depending on their design condition) through the rectangular aperture and manipulating the test object which the experimenter had placed at the base of the apparatus. Each subject then removed his hand(s) from the aperture, turned 90 deg right to face the identification card, and made his response by pointing to a figure. The duration of the procedure was determined by the individual subject, and the experimenter was careful not to rush the young subject's judgments. Each subject was allowed to use the hand of his choice (preferred hand) as long as he used the same hand on all single-hand trials.

A different randomized sequence of the 10 forms was presented to each subject. When all 10 forms had been presented, the subject repeated the task using two hands if one was used during the first testing and vice versa. Again the forms were presented in a different random order. The responses recorded were those which the subject made by pointing to a particular figure. No verbal responses were recorded, due to the relative inability of the subjects to verbally identify the figures.

The dependent variable in this study was the number of recognition errors made, with the maximum error score of 10 for each subject on each test. All forms were presented twice, with the entire experimental procedure requiring about $20 \mathrm{~min}$. 


\section{RESULTS}

The number of errors in haptic recognition was submitted to a 3 by 2 by 2 by 2 analysis of variance (dominance, order-of-tasks, hands, tests) with repeated measures on the tests factor. Results of this analysis indicated that there were significant differences in recognition performance related to subject's scaled dominance $[\mathrm{F}(2,70)=22.20$, $\mathrm{p}<.001]$, number of tests given $[\mathrm{F}(1,70)=55.46$, $\mathrm{p}<.001]$, and the interaction between the dominance and tests factors $[F(2,70=22.22, p<.001]$. Neither main effect due to order-of-tasks or due to hands was significant.

A plot of the Dominance by Tests interaction is given in Figure 1, where it can be seen that the form-dominant group made fewer errors than both the color-dominant group and the mixed group on each test. Application of Newman-Keuls test to the means on Test 1 indicated that the form group made significantly fewer errors than the color group $(\mathrm{p}<.001)$, which in turn made fewer errors than the mixed group $(p<.001)$. Application of the Newman-Keuls test to the means on Test 2 revealed that the rank order of the means had changed significantly between tests. The form dominant group still made fewer errors than the mixed group ( $p<.025)$; but on Test 2 , the mixed group made fewer errors than the color group $(p<.025)$. Examination of simple main effects of tests for each dominance group showed that only the mixed group significantly reduced the number of errors between tests $[F(1,70)=47.02, p<.001]$. The form and color groups showed nonsignificant changes between Test 1 and Test 2 ( $p>.10$ in both cases).

There were also significant interactions between hands and tests $[F(1.70)=23.49 . p<.001]$ and between dominance, hands. order of tasks, and tests $[F(2,70)=5.45, p<.01]$. Since the four-way interaction only accounted for $5 \%$ of the repeated measures variance, it was not dealt with further. The Hands by Tests interaction arose from the fact that the mean errors for subjects tested with one hand on Test $1(M=3.28)$ decreased significantly when they were tested with two hands on Test $2(M=1.71)$ $[F(1.70)=41.92, p<.001]$. Alternatively, subjects tested with two hands on Test $1(M=2.35)$ showed negligible change in errors when tested with one hand on Test $2(\mathrm{M}=2.74)[\mathrm{F}(1,70)=2.59, \mathrm{p}>.10]$.

Although the criterion used to assess the subjects' dominant dimension was fairly stringent $(87.5 \%)$, inspection of the raw data indicates that only 2 of the 26 mixed subjects would have been classified as having a dominant dimension if a lower criterion of $75 \%$ had been set.

\section{DISCUSSION}

As expected, a hierarchy of dimensional dominance was found among preschool children, which is a good predictor of performance on a haptic recognition task. Compared to subjects

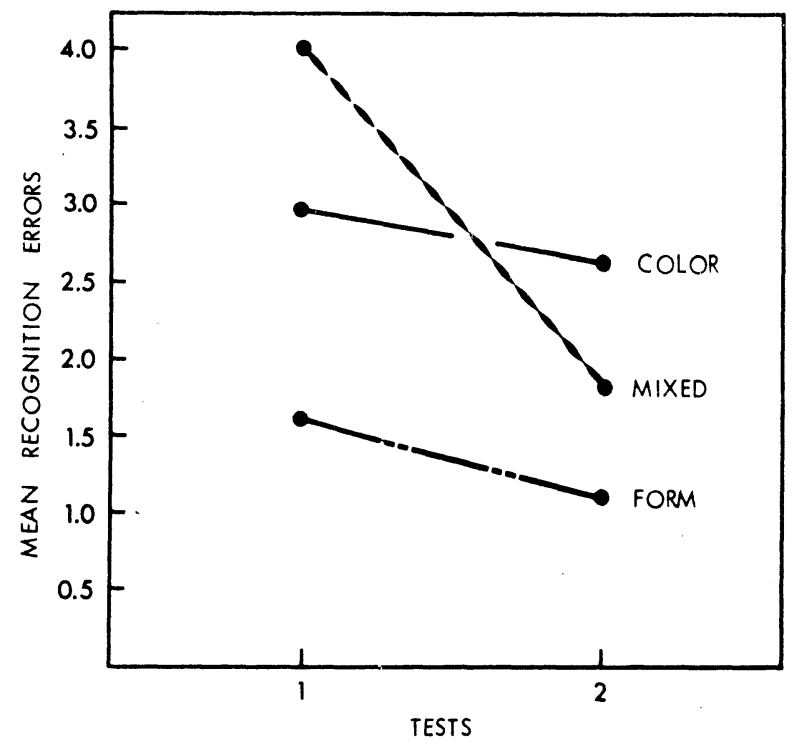

Figure 1. Mean errors on Test 1 and Test 2 for Dominance Groups by Tests interaction.

who were not form dominant, the form dominant subjects made fewer errors. which may be due both to their dominant dimension being relevant to the haptic task and a higher level of cognitive ability. If the color-dominant group can be considered developmentally inferior to the form group in cognitive or problem-solving ability, as suggested by Brian \& Goodenough (1929). Seitz \& Weir (1971), and others, then the present study demonstrates that this difference extends to haptic form recognition.

the significant improvement of mixed responders on Test 2 indicates that they were influenced by brief practice. This finding supports the hypothesis proposed by Suchman and Trabasso (1966) that multidimensional responding occurs when subjects are in a temporary transition stage, moving from color to form dominance. Although the improvement of mixed subjects on Test 2 was expected, their inferior performance on Test 1 was not. The Suchman and Trabasso transition hypothesis would place the mixed group inferiror to the form group but superior to the color group. One explanation of the performance of the mixed group would be to assume that they are subjects who have a short attention span or difficulty following direction. While they gave poor performance on the first haptic test, with practice their attention and ability to follow instructions may improve, resulting in fewer errors on the second test. A goal of further study in this area would be to obtain more information about mixed subjects perhaps by repeatedly scaling them, using a variety of scaling procedures, and testing when the experimenter has established extensive rapport with the subjects.

Tighe, et al.(1970) raised the question of the role of dominance scaling and its effect upon other tasks. They found that the dominant dimension produced faster discrimination shifts if the scaling test was administered prior to the discrimination tasks but not if it was administered after the discrimination tasks. The present study also included the order-of-testing control, with half the subjects being given the haptic task before dominance scaling and half afterwards. However, the present data indicated no effects due to order of task presentation. The results reported by Tighe et al. (1970) may not be replicable or simply may not generalize to a haptic recognition task.

\section{REFERENCES}

Brian. C. R., \& Goodenough, F. L. Relative potency of color and form perception at various ages. Journal of Experimental Psychology. 1929. 12. 197-213. 
Doenring, D. G. Color-form attitudes of deaf children. Journal of Speech and Hearing Research, 1960, 3, 242-248.

FERnANDEZ, F. Induced dimensional set and concept learning. Bulletin of the Psychonomic Society, 1973, 1, 261-263.

Kagan, J., \& Lemkin, J. Form, color, and size in children's conceptual behavior. Child Development, 1961, 32, 25-28.

Odom, R. D., \& Guzman, R. D. Development of hierarchies of dimensional salience. Developmental Psychology, 1972, 6, 271-287.

SEITZ, V., \& WEIR, M. W. Strength of dimensional preferences as a predictor of nursery-school children's performance on a concept-shift task. Journal of Experimental Child Psychology, 1971. 12, 370-386.
SMILEY, S. S., \& WeIR, M. W. The role of dimensional dominance in reversal and nonreversal shift behavior. Journal of Experimental Child Psychology, 1966, 4, 296-307.

SPREEN, O., \& GADDEs, W. H. Developmental norms for 15 neuropsychological tests age 6 to 15. Cortex, 1969, 5, 170-191.

Suchman, R.. \& Trabasso, T. Color and form preference in young children. Journal of Experimental Child Psychology, 1966, 3, 177-187.(a)

Tighe, L. J., Tighe, T. J., Waterhouse, M. D., \& Vasta, R. Dimensional preference and discrimination shift learning in children. Child Development, 1970, 41, 737-746.

(Received for publication September 3, 1975.) 